\title{
0 trabalho de campo como dispositivo de ensino, pesquisa e extensão na graduação de Medicina e Odontologia*
}

\section{Introdução}

A aprovação das diretrizes curriculares nacionais (DCNs) para a área da saúde, entre 2001 e $2002^{6}$, representou importante conquista para a Reforma Sanitária Brasileira, e tem orientado o processo de reformulação curricular nas instituições de Ensino Superior. As DCNs visam romper com a concepção de formação de profissionais de saúde baseada no modelo da medicina científica, que privilegia a abordagem centrada no hospital e em procedimentos, e se relaciona com um modelo pedagógico baseado em conteúdos, dissociados em áreas básicas e clínicas, e no incentivo à especialização precoce (Ceccim, Feuerwerker, 2004).

O perfil definido pelas DCNs para o profissional de medicina e odontologia (Brasil, 2002, 2001) é o de generalista, preparado para: compreender o processo saúde-doença nas suas múltiplas determinações, analisar problemas de saúde e buscar soluções para os mesmos. As DCNs enfatizam a importância do trabalho em equipe multiprofissional e a integralidade de práticas na rede de atenção à saúde; orientam os Projetos Pedagógicos dos Cursos (PPC) e consideram aluno como sujeito e professor como mediador do processo ensino-aprendizagem; e buscam uma formação integral - incluindo aspectos técnicos e éticos -, por meio da articulação entre ensino, pesquisa, extensão e assistência.

Os PPCs são construídos mediante as especificidades de sua área de atuação e devem ser elaborados em consonância com o Projeto Pedagógico Institucional (PPI). Este explicita o posicionamento da instituição a respeito do ser humano, do mundo e de sua relação na educação; pode representar um instrumento de ação política e pedagógica (Veiga, 2004) numa conjuntura globalizada e tecnológica; e explicitar, simultaneamente, o papel da instituição e sua contribuição social nos âmbitos local, regional e nacional (Brasil, 2006).

Um importante elemento constitutivo do PPC é o currículo, concebido como espaço de formação plural, dinâmico e multicultural, fundamentado em referenciais socioantropológicos, psicológicos, epistemológicos e pedagógicos.

O Instituto de Saúde da Comunidade (ISC), uma unidade de formação da Universidade Federal Fluminense (UFF), assumiu esta perspectiva pedagógica desde 1994, e atua em diversos cursos na perspectiva crítica do ensinoaprendizado na área de Saúde Coletiva. Participou, ativamente, dos processos de

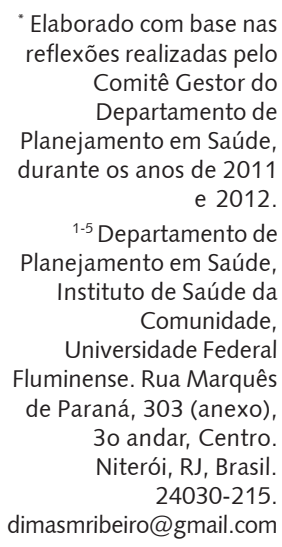

" Elaborado com base nas reflexões realizadas pelo Comitê Gestor do Departamento de Planejamento em Saúde durante os anos de 2011 e 2012.

${ }^{1-5}$ Departamento de Planejamento em Saúde, Instituto de Saúde da Comunidade Universidade Federal Fluminense. Rua Marquês de Paraná, 303 (anexo), 30 andar, Centro. Niterói, RJ, Brasil. 24030-215. dimasmribeiro@gmail.com

${ }^{6}$ Exceto medicina veterinária, psicologia, educação física e serviço social. 
reformulação curricular dos cursos de Medicina e Odontologia, e orienta todo o cotidiano de investigação e práticas no campo da Saúde Coletiva desenvolvido por estes cursos.

No curso de Medicina da UFF, a implantação do novo currículo ${ }^{7}$ contempla essas recomendações das DCNs. Sua implantação ocorreu num contexto histórico de articulação ensino-serviço-comunidade: a inserção da universidade na rede de serviços de saúde de Niterói foi fundamental para viabilizar a nova proposta curricular, e se constituiu numa "oportunidade de ampliar os cenários de práticas docentes, aproximando-se da realidade dos serviços e das populações" (March et al., 2006, p.298). Cabe considerar que a implementação do Sistema Único de Saúde (SUS) em Niterói, ao valorizar " a territorialização, a adscrição de clientela, a vinculação profissional/população, a hierarquização, a visão intersetorial, a promoção e prevenção da saúde e o controle social", tem "fomentado a mudança da formação profissional, para construir uma nova lógica de atenção à saúde" (March et al., 2006, p.298).

No curso de Odontologia, o PPC encontra-se em fase de implantação desde 2010. Após dez anos de discussão, a comunidade acadêmica delineou uma organização curricular capaz de favorecer o desenvolvimento de competências caracterizadas pelo vivenciar - refletir - decidir - agir, incorporando espaços integradores de aprendizagem. Trabalha-se na perspectiva da aproximação com a realidade do mundo do trabalho, salientando os múltiplos olhares sobre a realidade social e buscando o equilíbrio entre vocação técnico-científica e prática humanística.

Neste texto descrevemos a proposta de Trabalho de Campo (TC) desenvolvida pelo ISC como dispositivo de ensino, pesquisa e extensão 8 para professores/ alunos de medicina e odontologia. Está fundamentada na teoria de desenvolvimento humano e numa pedagogia que defende que as oportunidades oferecidas na Educação Superior, para o desenvolvimento das capacidades dos estudantes como pessoas éticas, têm importância fundamental para a promoção da cidadania do aluno (Walker, 2006).

\section{Pressupostos teóricos}

Pedro Demo (2001) argumenta que as dimensões 'ensino', 'pesquisa' e 'extensão' do trabalho acadêmico poderiam ser reduzidas a duas: "reconstruir o conhecimento" e "educar novas gerações", apreendidas sob os termos de 'extensão' ao 'ensino' e à 'pesquisa'. Esses termos sugerem que o compromisso social com a promoção da justiça, dos direitos humanos e da cidadania geralmente relacionado às ações extensionistas - permanece como algo separado, voluntário e intermitente, quando deveria ser intrínseco à organização curricular (Demo, 2001). Numa perspectiva semelhante, Boaventura dos Santos (2005) concebe 'extensão' como

modo alternativo ao capitalismo global, atribuindo às universidades uma participação ativa na construção da coesão social, no aprofundamento da democracia, na luta contra a exclusão social e a degradação ambiental, na defesa da diversidade cultural. (Santos, 2005, p.73)

A universidade tem função determinante na formação de profissionais outrora chamados 'recursos humanos': seja por demonstrar eficácia em comparação com
7 O novo currículo tem como pressuposto a "constituição de um perfil profissional [...] adequado à realidade sanitária local, ao mercado de trabalho e ao desenvolvimento científico e tecnológico vigente", para formar profissionais humanistas, capazes de apresentar comportamento ético e habilidades psicomotoras específicas do trabalho médico (Saippa-Oliveira, Koifman, Marins, 2004, p.310).
${ }^{8}$ Neste artigo usamos a denominação Trabalho de Campo (TC) para ressaltar os aspectos comuns destas

disciplinas, caracterizadas por inserir alunos de graduação, desde o início de sua formação, no âmbito das organizações do Estado (setor saúde e outros setores), da sociedade civil e organizações sociais não governamentais. 


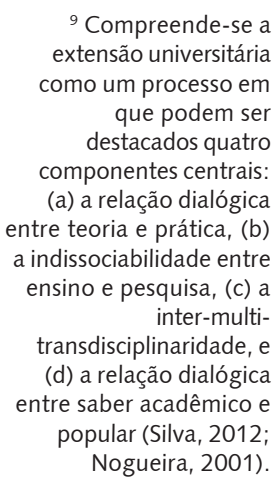

${ }^{10}$ Para um pensamento pós-abissal, Santos $(2010$ p.54) propõe a "ecologia dos saberes" que "tem como premissa a ideia da diversidade epistemológica do mundo", onde convive uma "pluralidade de formas de conhecimento além do conhecimento científico". Não pretende negar o valor do conhecimento científico, mas "explorar a pluralidade interna da ciência" e "promover a interação e a interdependência entre os saberes científicos e outros saberes, não científicos" (Santos, 2010, p.57). instituições de outra natureza, seja pelo papel único na definição da ética de desenvolvimento nacional e como lócus privilegiado de crítica e transformação social. Isto se dá pela atuação no ensino, na participação social a partir da extensão, e na criação do novo como resultado do processo de pesquisa científica (Koifman, 2011) ${ }^{9}$.

Para Edgar Morin (2004a), os problemas de nossa "era planetária" tornam-se cada vez mais complexos e globalizados, exigindo reforma do pensamento capaz de encontrar soluções que deem conta de sua multidimensionalidade. Para o mesmo autor, apenas um "pensamento complexo capaz de ligar, contextualizar e globalizar", operando de maneira transdisciplinar, é "capaz de prolongar-se numa ética da interdependência entre os seres humanos", favorecendo o "sentido da responsabilidade e da cidadania" (Morin, 2004b, p.22, 27). Esta reforma do pensamento é, para o autor,

uma necessidade democrática fundamental: formar cidadãos capazes de enfrentar os problemas de sua época é frear o enfraquecimento democrático que suscita, em todas as áreas políticas, a expansão da autoridade dos experts, especialistas de toda ordem, que restringem progressivamente a competência dos cidadãos. (Morin, 2004a, p.103)

Santos (2010, p.32), por outro lado, sustenta que "o pensamento moderno ocidental é um pensamento abissal", consistindo num "sistema de distinções visíveis e invisíveis" que divide "a realidade social em dois universos distintos: o universo 'deste lado da linha' e o universo 'do outro lado da linha', de modo que 'o outro lado da linha' desaparece enquanto realidade", sendo "produzido como inexistente". Esta distinção, na avaliação do autor, se funda em outra, "entre as sociedades metropolitanas e os territórios coloniais".

Do ponto de vista da produção do conhecimento, "o pensamento abissal consiste na concessão à ciência moderna do monopólio da distinção entre o verdadeiro e o falso", tornando inexistente os conhecimentos produzidos "do outro lado da linha" - conhecimentos populares, leigos, camponeses ou indígenas etc.- , considerados "crenças, opiniões, magia, idolatria, entendimentos intuitivos ou subjetivos, que, na melhor das hipóteses, podem tornar-se objetos ou matériaprima para a inquirição científica" (Santos, 2010, p.34). Assim, converte este lado da linha em sujeito do conhecimento, e o outro lado da linha em objeto do conhecimento ${ }^{10}$.

Mora-Osejo e Borda (2006, p.715) enfatizam a necessidade de se desenvolverem "paradigmas científicos e quadros técnicos de referência" para entendimento das realidades locais. Disto podem "surgir descobertas e iniciativas úteis para a sociedade local, que minorem as crises do próprio contexto", de modo que, deste processo, "resultem valores partilhados, geradores de solidariedades" e fortalecedores da identidade cultural (Mora-Osejo, Borda, 2006, p.718).

Nesta perspectiva, "deve dar-se preferência às formas de conhecimento que garantam a maior participação dos grupos sociais envolvidos na concepção, na execução" e no controle da intervenção, favorecendo-se parcerias da universidade com: organizações não governamentais, movimentos sociais, grupos sociais vulneráveis e grupos sociais populares, entre outros (Santos, 2010, p.60). Para Santos (2010), a justiça social global implica justiça cognitiva capaz de romper com o pensamento abissal.

Adota-se, neste texto, uma pedagogia da educação superior com foco nas capacidades e funcionamentos básicos, distinta da Abordagem das Capacitações 
(capability), formuladas por Nussbaum e Sen, embora seguindo a tradição instaurada por estes autores (Dias, 2009). O desenvolvimento humano concebido como promoção de capacidades e funcionamentos fundamentais requer condições materiais de existência - econômicas, sociais, culturais etc. - para serem efetivamente exercidos.

Tais capacidades seriam essenciais nas diferentes esferas onde os seres humanos atuam (saúde, trabalho, educação, lazer, política etc.) e nos vários estágios de um ciclo de existência (nascimento, infância, adolescência, fase adulta, velhice e morte) (Nussbaum, 2000).

Como capacidades centrais, Nussbaum (2000) reconhece: a liberdade de ser saudável, viver uma vida com longevidade normal, exercer controle sobre o ambiente, estar bem nutrido e abrigado, não ser acometido por doenças evitáveis ou morrer prematuramente; ter integridade corporal, exercer a razão prática, pensar e imaginar, vivenciar emoções e estabelecer relações com os outros, entre outras.

Capacitações, capacidades e funcionamentos, segundo Sen, são conceitos correlacionados, porém distintos. Capacitações (capability) correspondem à liberdade de realizar ou alcançar uma combinação de funcionamentos que expressem oportunidades reais para realizar modos de viver, dentro de um leque de alternativas disponíveis. Capacidades se referem às várias combinações de estados e ações inter-relacionados em que uma pessoa pode estar ou que pode realizar. Funcionamentos referem-se a cada ação ou estado que compõem diversas opções de combinações disponíveis.

Walker (2006) propõe uma pedagogia da Educação Superior baseada na Abordagem das Capacitações, concebendo a pedagogia como método de ensino (envolve a relação entre professores e alunos e as condições estruturais que possibilitam a aprendizagem) e como projeto ético (em que seus propósitos envolvem valores éticos e o desenvolvimento de conhecimentos e habilidades). Desenvolve uma metodologia para a seleção de uma lista de capacidades a serem estimuladas com os estudantes e como parâmetro para avaliar a aprendizagem de cada um. Inclui: "razão prática" - capacidade de fazer escolhas refletidas e socialmente responsáveis; "relações e redes sociais" - capacidade de participar em grupos para uma aprendizagem colaborativa; "respeito, dignidade e reconhecimento" - capacidade de respeitar e ser respeitado; "resistência educacional" - capacidade de governar seus estudos -, entre outras.

\section{Capacidades/funcionamentos e TC}

Nossa discussão baseia-se em experiências desenvolvidas nas disciplinas de Trabalho de Campo Supervisionado 1 e 2 do curso de Medicina (TCS 1 e 2), e de Saúde Bucal Coletiva 3 e Estágio Supervisionado 1 do curso de Odontologia (SBC 3 e ES 1). O corpo docente é composto por profissionais com mestrado e/ou doutorado na área de Saúde Coletiva.

Durante o ano de 2011, foram realizadas oficinas sobre o TC promovido pelo ISC-UFF. A sistematização das experiências e reflexões desenvolvidas por professores, técnicos e alunos monitores permitiu analisar os resultados em termos de capacidades e funcionamentos, conforme proposto neste trabalho.

O TC apoia-se numa pedagogia participativa e inclusiva, preocupada em promover capacidades voltadas ao desenvolvimento humano e uma sociedade justa e democrática.

\section{As disciplinas: breve descrição}

Nas disciplinas ofertadas ao curso de Medicina, os alunos são divididos em grupos entre oito e 12 alunos, para atividades teóricas e práticas, com a carga horária de nove horas semanais. Os alunos são preparados para entrar em campo, conhecer e observar distintas realidades socioculturais e interagir com vários atores (pacientes/usuários, profissionais de saúde, gestores, dirigentes etc.) em diferentes cenários, como: movimentos sociais, organizações não governamentais, unidades de saúde do SUS, entre outros. As disciplinas TCS 1 e 2 fazem parte do Programa Prático-Conceitual do curso de Medicina, tendo cada uma um ano de duração, a partir do primeiro período do curso. Enquanto TCS 1 fundamenta-se no reconhecimento do contexto de ação sociocultural do profissional de saúde, compreendendo "sua responsabilidade na investigação dos determinantes sociais da saúde e da doença, 
através da caracterização qualitativa e quantitativa do cenário ambiental, populacional e psicossocial, nos níveis local e municipal" (Saippa-Oliveira, Koifman, Marins, 2004, p.311), TCS 2 tem como objetivo reconhecer o perfil epidemiológico da população, analisar o funcionamento das unidades de saúde e o "impacto das ações programadas sobre as condições locais diagnosticadas pelos serviços de saúde" (Saippa-Oliveira, Koifman, Marins, 2004, p.313).

No curso de Odontologia, formam-se grupos de até seis alunos para discussões teóricas e atividades práticas, com a carga horária de seis horas semanais. O TC compreende conhecimento e aproximação com equipes e processos de trabalho em unidades de Saúde da Família no município de Niterói. O grupo aprimora projetos terapêuticos de famílias adscritas a partir do diagnóstico e da lógica de necessidades de saúde. O objetivo é alcançado por meio do: envolvimento com a dinâmica da unidade/módulo, acompanhamento de visitas domiciliares e estudo aprofundado dos instrumentos de registro (cadastro e prontuários), sempre envolvendo professores e equipes de saúde. Durante um semestre, cada grupo de alunos se dedica ao estudo do território, para: entender percursos e formas de acesso aos domicílios (incluindo áreas de risco), conhecer e potencializar aparelhos sociais (escolas, creches, associação de moradores, igrejas, organizações sociais, entre outros), na área de abrangência da policlínica regional que cobre o setor da equipe com a qual o grupo irá trabalhar. Ao final do semestre, após consolidarem todos os dados observados e situações vivenciadas, os alunos elaboram e operacionalizam, com a equipe, um plano de intervenção, sempre na perspectiva do ganho de autonomia por parte dos moradores/comunidade.

\section{Capacidades e funcionamentos no TC}

As capacidades e funcionamentos a serem promovidos pelo TC estão relacionados,

fundamentalmente, com alguns pressupostos do campo da Saúde Coletiva, conforme foi se delineando, no Brasil, a partir da década de 1970 (Paim, Almeida Filho, 2000). Entende-se este como um campo de natureza híbrida, com uma "lógica teórico-epistemológica de produção do conhecimento, seja ela interpretativa ou explicativa, dependendo da área em que se origina", e uma "lógica operativa e pragmática da eficácia, decorrente da intervenção normativa na ordem da vida, no sentido da erradicação ou controle do adoecimento coletivo" (Luz, 2009, p.306).

Desenvolve-se a partir de três disciplinas básicas - a epidemiologia, o planejamento em saúde e as ciências sociais em saúde - e opera, conforme o modelo, a interdisciplinariedade, a multidisciplinariedade ou a transdisciplinariedade (Luz, 2009). Constrói-se como modelo de produção do conhecimento que integra a produção acadêmica, nas suas várias modalidades, e, também, o "conhecimento gerado a partir da prática vivenciada pelas populações ou por usuários dos serviços (ou pacientes), superando a clivagem entre senso comum versus ciência, típica da modernidade" (Luz, 2009, p.309).

Paim e Almeida Filho (2000, p.60) caracterizam a Saúde Coletiva a partir de alguns pressupostos básicos: a saúde articulada à estrutura da sociedade por meio de suas instâncias econômica e políticoideológica, possuindo, portanto, uma historicidade; as ações de saúde (promoção, proteção, recuperação, reabilitação) como prática social, permeada por relações técnicas e sociais, e sujeitas às "influências do relacionamento dos grupos sociais"; o objeto da Saúde Coletiva construído nos limites entre biológico e social, e compreendendo a investigação dos determinantes da produção social das doenças e da organização dos serviços de saúde, e o estudo da historicidade do saber e das práticas sobre os mesmos.

Para que o TC possa operar como dispositivo de ensino-pesquisa-extensão e promover capacidades e funcionamentos como descritos acima, sua organização deverá estar baseada na aproximação com espaços/territórios específicos, como define Milton Santos (2009) e Monken e Barcellos (2005), e o processo ensino-aprendizagem estar conectado aos princípios da pesquisa-ação.

Pesquisa-ação é uma modalidade de pesquisa constituída por um ciclo básico - investigação de um problema, planejamento e execução de ações para resolvê-lo, avaliação de resultados destas ações e programação de mudanças necessárias para a resolução do problema (Tripp, 2005). Alguns princípios fundamentais para que o TC possa se desenvolver dentro desta perspectiva são: o compromisso político de busca pela justiça social e cidadania, para conhecer problemas comunitários e formular estratégias 
para enfrentá-los a partir da realidade vivida no cotidiano pelos indivíduos e coletividades (Thiollent, 2006); a relação entre investigador-educador e grupos populares, com o pressuposto de que todas as pessoas e todas as culturas são fontes originais de saber (Brandão, 2006); o relacionamento dinâmico entre teoria e prática (Demo, 2008); e a natureza formativa da metodologia - podendo-se falar no tripé pesquisa-formação-ação -, a impulsionar processos dialógicos de aprendizagem coletiva nos quais "o sujeito deve tomar consciência das transformações que vão ocorrendo em si próprio e no processo" (Franco, 2005, p.486).

Na proposta aqui apresentada, consideram-se dois ciclos de pesquisa-ação, dentro de uma determinada abrangência territorial: um corresponde aos cenários onde se desenvolvem práticas e saberes que operam na sociedade civil e nas políticas setoriais do Estado, não apenas no setor saúde; outro acontece em cenários do setor saúde, no nível primário ou secundário da rede de atenção.

\section{Primeiro ciclo}

Este ciclo conta com quatro fases: na primeira, os alunos têm preparação inicial para atividades práticas com estudo introdutório à pesquisa social, reconhecimento teórico do tema analisador ${ }^{11}$, busca do conhecimento das diversas abordagens e contextos, e mapeamento de locais a visitar. No eixo teórico-prático, discutem as relações entre condições de vida e determinantes econômicos, sociais, culturais do processo saúde-doença, por um lado, e as respostas, do Estado e da sociedade civil, a estas condições, por outro. São ressaltados diferentes grupos sociais e suas condições de vida específicas; utilizam-se dados quantitativos e qualitativos (primários e secundários) após consulta a bancos de dados, pesquisas, visitas, observação participante, entrevistas, entre outras possibilidades, durante todas as fases. Na segunda, os alunos exploram o território tendo, como eixo teórico-prático, a dimensão cultural do processo saúde-doença e grupos/ atores sociais da sociedade civil (igrejas, ONGs, movimentos sociais, grupos comunitários etc.). Reflete-se sobre: a relação entre cultura, políticas públicas e cidadania, a dicotomia saber médico versus popular, e as diversas racionalidades médicas. As redes de apoio social devem ser reconhecidas, bem como seu papel na promoção da cidadania. O eixo teórico é a integralidade em saúde. $\mathrm{Na}$ terceira, os alunos analisam a presença do Estado no território, considerando as políticas públicas setoriais. O eixo teórico-prático é a integralidade da atenção e a intersetorialidade das ações. Enfatizam-se dimensões culturais, sociais e políticas do ato de cuidar e de redes sociais de solidariedade. Na quarta, os alunos identificam um problema de saúde a ser trabalhado, com ênfase num dos polos do processo, pesquisa ou ação.

\section{Segundo ciclo}

Deve ser desenvolvido numa região, no nível primário (em especial) ou secundário da rede de saúde. Na quinta fase, os alunos são apresentados à área de abrangência de unidades de saúde de nível primário, e fazem o reconhecimento teórico-prático do território. Na sexta, são inseridos numa unidade de saúde primária e analisam seu funcionamento nos seus espaços/ações (recepção/pré-consulta, vacinação, pequenos procedimentos, consulta de enfermagem ou médica, farmácia, vigilância em saúde etc...); observam e problematizam o processo de trabalho dos profissionais da unidade e sua relação com a rede de atenção à saúde. O eixo teórico é formado pelos modelos

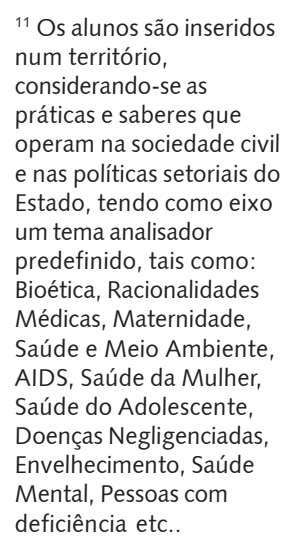


tecnoassistenciais, o processo de trabalho e o trabalho em equipe multiprofissional, com ênfase na promoção e proteção da saúde. Na sétima, os alunos são apresentados à comunidade em que estão inseridos por meio da unidade, e realizam diagnóstico de necessidades de saúde de famílias selecionadas, segundo: condições de vida, acesso às tecnologias, constituição de vínculo e grau de autonomia do usuário. Na última fase, constroem ou aprimoram projetos terapêuticos singulares com a equipe de saúde e desenham plano de intervenção, sendo, o eixo teórico, a produção do cuidado em saúde. Nessa fase, devem recorrer aos conceitos trabalhados nas fases anteriores, enfatizando a reflexão sobre estilos de vida, redes sociais de apoio e itinerário terapêutico.

\section{Considerações finais}

Neste trabalho, descrevemos e analisamos uma proposta pedagógica que aposta no trabalho de campo como dispositivo articulador entre ensino, pesquisa e extensão. As disciplinas apresentadas têm, como pressuposto geral: o processo de ensino-aprendizagem com metodologias ativas, uma relação dinâmica entre teoria e prática, propondo, ao estudante, a interação com o mundo do trabalho e a reflexão sobre a realidade vivenciada.

Tais disciplinas de campo atendem à necessidade que os estudantes têm, desde o início de sua graduação, de conhecer experiências dos cenários reais de trabalho, bem como questões de saúde que enfrentarão futuramente.

As disciplinas de campo, bem como suas atividades elaboradas para atender a essa demanda, vêm sendo analisadas e estudadas por pesquisas acadêmicas desde a iniciação científica até programas de mestrado, doutorado, e pós-doutorado. Ao mesmo tempo em que alunos e professores vivenciam, aprendem e pesquisam com base nesse cotidiano apresentado pelo TC, também respondem às necessidades de saúde dos cidadãos, a exemplo dos planos de intervenção que alimentam o Programa de Extensão "Diversificação de Cenários de Aprendizagem em Saúde - A integração Ensino-ServiçoSociedade"

Gomes (2011) conclui que toda esta experiência possibilita "formar médicos mais adaptados às demandas do SUS e que [...] conseguem perceber a importância de uma prática voltada para a integralidade" (Gomes, 2011, p.58). Nogueira (2012) analisa que "a partir dessas inovações, pode-se tentar traçar um perfil do médico formado: que conhece mais o SUS, é mais ético, possui maior capacidade crítica e reflexiva, tem mais abertura para trabalhar em equipe, [...]" (Nogueira, 2012, p.144).

Vários autores (Nogueira, 2012; Gomes, 2011; Saippa-Oliveira, 2010; Fernandez, 2009; Pontes, 2005; Koifman, 2002, 1996) demonstram que o formato da disciplina de campo descrito neste artigo aproxima-se da recomendação estabelecida nas Diretrizes Curriculares, para a formação de um profissional reflexivo e humano.

A ação formativa na saúde impõe constantes desafios para se construírem redes de aprendizagem docente sob o eixo da integralidade das ações de saúde. Referimo-nos ao processo pedagógico desenvolvido em múltiplos cenários, priorizando situações reais. A metodologia é centrada no compartilhamento de experiências e vivências, por intermédio de supervisão dialogada, na busca de mudanças institucionais, apropriação ativa de saberes, fortalecimento das ações em equipe, e produção de uma nova maneira de se organizar o trabalho em saúde (Saippa-Oliveira, Fernandez, Koifman, 2010).

Na proposta descrita, muitas vezes, os docentes são desafiados a reformular os princípios gerais dos ciclos e fases descritos, a partir das experiências vivenciadas, discutidas e sistematizadas coletivamente, o que, por sua vez, propicia novas sínteses a partir dos contextos nos quais o processo ocorre.

Esse movimento é uma inovação para docentes do Ensino Superior, acostumados a processos de planejamento, execução e avaliação das suas atividades de forma individual e isolada. Superar essa forma de atuação em nome de um processo coletivo possibilita conhecimento entre os pares e capacita para o enfrentamento de alterações previsíveis ou imprevisíveis.

Consideramos, por fim, que o avanço na formação em saúde dar-se-á em processos concomitantes de desenvolvimento pessoal e institucional, desafio a ser considerado na proposição político-pedagógica dos cursos de graduação em saúde. 


\section{Colaboradores}

Todos os autores trabalharam na concepção, delineamento e redação do artigo, construíram e revisaram a versão apresentada.

\section{Referências}

BRANDÃO, C.R. A pesquisa participante e a participação da pesquisa: um olhar entre tempos e espaços a partir da América Latina. In: BRANDÃO, C.R.; STRECK, D.R. (Orgs.). Pesquisa participante: a partilha do saber. Aparecida: Ideias e Letras, 2006. p.21-54.

BRASIL. Ministério da Educação e Cultura, Comissão Nacional de Avaliação da Educação Superior, Instituto de Pesquisa Econômica Aplicada. Avaliação externa de instituições de educação superior. Brasília: MEC, 2006.

Conselho Nacional de Educação. Câmera de Educação Superior. Resolução $\overline{\mathrm{CNE} \backslash \mathrm{CES}} \mathrm{n}^{\circ} 4$, de 7 de novembro de 2001. Diretrizes Curriculares Nacionais do Curso de Graduação em Medicina. Diário Oficial da União, Brasília, DF, 4 mar. 2002. Seção 1 , p.10.

Conselho Nacional de Educação. Câmera de Educação Superior. Resolução CNE \CES n³, de 19 de fevereiro de 2002. Diretrizes Curriculares Nacionais do Curso de Graduação em Odontologia. Diário Oficial da União, Brasília, DF, 9 nov. 2001. Seção 1, p.38.

CECCIM, R.B.; FEUERWERKER, L.C.M. Mudança na graduação das profissões de saúde sob o eixo da integralidade. Cad. Saude Publica, v.20, n.5, p.1400-10, 2004.

$\mathrm{DEMO}, \mathrm{P}$. Pesquisa participante: saber pensar e intervir juntos. 2.ed. Brasília: Líber Livro Editora, 2008.

O lugar da extensão. In: FARIA D.S. (Org.). Construção conceitual da extensão universitária na América Latina. Brasília: Universidade de Brasília, 2001. p.141-58.

DIAS, M.C. O "bom governo": diretrizes de governo em uma democracia. Diversitates, V.1, n.1, p.77-87, 2009.

FERNANDEZ, V.S. Práticas Integradas na formação em saúde: desafios e possibilidades de transformação das práticas pedagógicas no curso de Nutrição da UFF. 2009. Dissertação (Mestrado) - Escola Nacional de Saúde Pública, Rio de Janeiro. 2009.

FRANCO, M.A.S. Pedagogia da pesquisa-ação. Educ. Pesqui., v.31, n.3, p.483-502, 2005.

GOMES, L.N.A. Integralidade pelos alunos do Internato em Clínica Médica da UFF. 2011. Dissertação (Mestrado) - Universidade Federal Fluminense, Niterói. 2011.

KOIFMAN, L. A função da universidade e a formação médica. Rev. Bras. Educ. Med., v.35, n.2, p.145-6, 2011.

O ensino médico no Brasil e na Argentina: uma abordagem comparativa. 2002. Tese (Doutorado) - Escola Nacional de Saúde Pública, Rio de Janeiro. 2002.

. A crítica ao modelo biomédico na reformulação curricular do curso de medicina da Universidade Federal Fluminense. 1996. Dissertação (Mestrado) - Escola Nacional de Saúde Pública, Rio de Janeiro. 1996.

LUZ, M.T. Complexidade do campo da saúde coletiva: multidisciplinariedade, interdisciplinariedade e transdiciplinaridade de saberes e práticas: análise sócio-histórica de uma trajetória paradigmática. Saude Soc., v.18, n.2, p.304-11, 2009. 
$M A R C H, C$. et al. O currículo de medicina da Universidade Federal Fluminense: revisitando uma experiência. In: PINHEIRO, R.; MATTOS, R.A. (Orgs.). Ensinar Saúde: a integralidade e o SUS nos cursos de graduação na área de saúde. Rio de Janeiro: IMS/ UERJ, Cepesc, Abrasco, 2006. p.295-309.

MONKEN, M.; BARCELLOS, C. Vigilância em saúde e território utilizado: possibilidades teóricas e metodológicas. Cad. Saude Publica, v.21, n.3, p.898-906, 2005.

MORA-OSEJO, L.E.; BORDA, O.F. A superação do Eurocentrismo: enriquecimento do saber sistêmico e endógeno sobre o nosso contexto tropical. In: SANTOS, B.S. (Org.). Conhecimento prudente para uma vida decente: um discurso sobre as ciências revisitado. 2.ed. São Paulo: Cortez, 2006. p.711-20.

MORIN, E. Cabeça bem-feita: repensar a reforma, reformar o pensamento. 10.ed. Rio de Janeiro: Bertrand Brasil, 2004a.

Educação e complexidade: os setes saberes e outros ensaios. 2.ed. revista. São Paulo: Cortez, 2004b.

NOGUEIRA, M.D.P. Extensão universitária no Brasil: uma revisão conceitual. In: FARIA, D.S. (Org.). Construção conceitual da extensão universitária na América Latina. Brasília: Universidade de Brasília, 2001. p.57-72.

NOGUEIRA, M.I. Retratos da formação médica nos novos cenários de prática. Rio de Janeiro: Hucitec, 2012.

NUSSBAUM, M.C. Women and human development: the capabilities approach. Cambridge: Cambridge University Press, 2000.

PAIM, J.S.; ALMEIDA FILHO, N. A crise da saúde pública e a utopia da saúde coletiva. Salvador: Casa da Qualidade Editora, 2000.

PONTES, A.L. Saber e prática docente na transformação do ensino médico: reflexões a partir da fala de preceptores do curso de medicina da UFF. 2005. Dissertação (Mestrado) - Escola Nacional de Saúde Pública, Rio de Janeiro. 2005.

SAIPPA-OLIVEIRA, G. Saberes e esquemas de ação docente em saúde coletiva. 2010. Tese (Doutorado) - Escola Nacional de Saúde Pública, Rio de Janeiro. 2010.

SAIPPA-OLIVEIRA, G.; FERNANDEZ, V.S.; KOIFMAN, L. Trabalho e formação: diálogos necessários para a construção de práticas do cuidado. In: PINHEIRO, R.; SILVA JÚNIOR, A.G. (Orgs.). 10 anos do Projeto Intregralidade: por uma sociedade cuidadora. Rio de Janeiro: Cepesc-IMS-UERJ, Abrasco, 2010. p.297-312.

SAIPPA-OLIVEIRA, G.; KOIFMAN, L.; MARINS, J.J.N. A busca da integralidade nas práticas de saúde e a diversificação dos cenários de aprendizagem: o direcionamento do curso de medicina da UFF. In: PINHEIRO, R.; MATTOS R.A. (Orgs.). Cuidado: as fronteiras da integralidade. Rio de Janeiro, RJ: Hucitec/Abrasco, 2004. p.307-19.

SANTOS, B.S. A universidade no século XXI: para uma reforma democrática e emancipatória da universidade. 2.ed. São Paulo: Cortez, 2005.

Para além do pensamento abissal: das linhas globais a uma ecologia de saberes. In: SANTOS, B.S.; MENEZES, M.P. (Orgs.). Epistemologias do sul. São Paulo: Cortez, 2010. p.31-83.

SANTOS, M. A natureza do espaço: técnica e tempo, razão e emoção. 4.ed. São Paulo: Editora da Universidade de São Paulo, 2009.

SILVA, A.F.L. Extensão universitária na UFF: uma análise crítica no campo da saúde com foco na formação. 2012. Dissertação (Mestrado) - Instituto de Saúde da Comunidade, Universidade Federal Fluminense, Niterói. 2012. 
O TRABALHO DE CAMPO COMO DISPOSITIVO $\ldots$

THIOLENT, M. A inserção da pesquisa-ação no contexto da extensão universitária. In: BRANDÃO, C.R.; STRECK, D.R. (Orgs.). Pesquisa participante: a partilha do saber. Aparecida: Ideias e Letras, 2006. p.151-65.

TRIPP, D. Pesquisa-ação: uma introdução metodológica. Educ. Pesqui., v.31, n.3, p.443-66, 2005

VEIGA, I.P.A. (Org.) Projeto político-pedagógico da escola: uma construção possível. Campinas: Papirus, 1995.

WALKER, M. Higher education pedagogies: a capabilities approach. Berkshire: Open University Press, 2006. 
Descreve-se a proposta pedagógica de disciplinas nos cursos de Medicina e Odontologia da Universidade Federal Fluminense (UFF), Rio de Janeiro, Brasil, tendo o trabalho de campo como dispositivo de articulação entre ensino, pesquisa e extensão; e, como pressuposto geral, o processo de ensino-aprendizagem baseado em: metodologias ativas, a relação dinâmica entre teoria e prática, e a proposição, ao estudante, de interação com o mundo do trabalho e reflexão sobre a realidade vivenciada. Espera-se contribuir para o debate na área de formação em saúde, em especial no campo da Saúde Coletiva, com base na reformulação determinada pelas Diretrizes Curriculares Nacionais aprovadas em 2001/2002. E, também, para a superação das formas tradicionais de atuar por outras que possibilitem conhecimento entre os pares e capacitação para o enfrentamento de alterações previsíveis ou imprevisíveis no cotidiano das escolas de formação em saúde.

Palavras-chave: Formação de recursos humanos. Sistema Único de Saúde. Currículo. Assistência integral à saúde.

Fieldwork as a teaching, research and extension device in undergraduate Medicine and Dentistry

This study describes the pedagogical proposals of disciplines within the medicine and dentistry courses at Universidade Federal Fluminense (UFF). Fieldwork has been backed as a means for linking teaching, research and extension, under the general assumption of a learning-teaching process based on active methodologies, a dynamic relationship between theory and practice, and proposals enabling students to interact with the world of work and reflect on the realities experienced. We hope to contribute towards the debate on healthcare training, especially with regard to the field of public health, consequent to the curriculum reformulation determined by the National Curriculum Guidelines (approved in 2001/2002). We also hope to contribute towards transforming traditional methods into other ways of acting that enable knowledge among peers and capacitation to cope with foreseeable or unforeseeable changes to the routine of healthcare training schools.

Keywords: Human resources formation. Brazilian National Health System. Curriculum. Comprehensive healthcare.

\section{El trabajo de campo como un dispositivo de enseñanza, investigación y extensión en los cursos de Medicina y Odontología}

El artículo describe la propuesta pedagógica de asignaturas en los cursos de Medicina y Odontología en la Universidad Federal Fluminense, Río de Janeiro, Brasil, considerando el trabajo de campo como dispositivo de articulación entre enseñanza, investigación y extensión y como base general del proceso de enseñanza-aprendizaje basado en metodologías activas, la relación dinámica entre teoría y práctica y la propuesta al estudiante de la interacción con el mundo del trabajo y la reflexión sobre la realidad vivida. Se espera contribuir con el debate en el área de Formación en Salud, en especial en el campo de Salud Colectiva, a partir de la reformulación determinada por las Directrices Curriculares Nacionales aprobadas en 2001/2002. También se espera contribuir para superar las formas tradicionales de actuación, proporcionando otras que posibiliten el conocimiento entre los pares y la capacitación para el enfrentamiento de situaciones previsibles e imprevisibles en el cotidiano de las escuelas de formación en salud.

Palabras clave: Formación de recursos humanos. Sistema Único de Salud. Currículo. Atención integral a la salud. 
